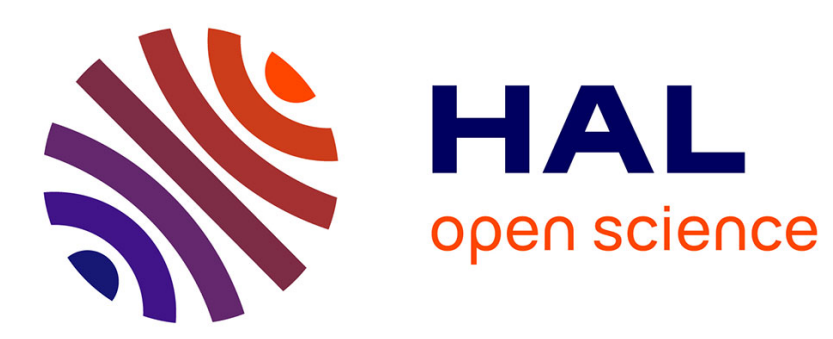

\title{
Channel Modeling in a Phonon-based Quantum Network for Nano-communications
}

\author{
Valeria Loscrì, Anna Maria Vegni
}

\section{To cite this version:}

Valeria Loscrì, Anna Maria Vegni. Channel Modeling in a Phonon-based Quantum Network for Nanocommunications. NanoCom, ACM, Sep 2016, New York, United States. 10.1145/2967446.2967480 . hal-01345403

\section{HAL Id: hal-01345403 \\ https://hal.inria.fr/hal-01345403}

Submitted on 13 Jul 2016

HAL is a multi-disciplinary open access archive for the deposit and dissemination of scientific research documents, whether they are published or not. The documents may come from teaching and research institutions in France or abroad, or from public or private research centers.
L'archive ouverte pluridisciplinaire HAL, est destinée au dépôt et à la diffusion de documents scientifiques de niveau recherche, publiés ou non, émanant des établissements d'enseignement et de recherche français ou étrangers, des laboratoires publics ou privés. 


\section{Channel Modeling in a Phonon-based Quantum Network for Nano-communications}

\author{
Valeria Loscrí \\ Inria Lille-Nord Europe \\ FUN Research team \\ Lille, France \\ valeria.loscri@inria.fr
}

\author{
Anna Maria Vegni \\ Department of Engineering \\ COMLAB Telecommunication laboratory \\ Roma Tre University, Rome, Italy \\ annamaria.vegni@uniroma3.it
}

\begin{abstract}
Quantum phenomena represent a natural direction for developing nanotechnology, since it has to be considered as a new scale where new phenomena can occur and can be exploited for information purpose. Specific quantum particles are phonons, the quanta of mechanical vibrations (i.e., acoustic excitations), that can be analyzed as potential information carriers in a body network context.

In this paper we derive the theoretical expression of the channel capacity of a phonon-based quantum network. By irradiating a sample of biological human tissue with an electromagnetic field, we obtain the generation of phonons from photon-phonon interaction, and can observe the information capacity in the frequency range $\left[10^{3}-10^{12}\right] \mathrm{Hz}$.
\end{abstract}

\section{Keywords}

Quantum computing, phonons, nano-communications, information capacity

\section{INTRODUCTION}

Traditional communication paradigms are not suitable in the context of nanotechnology, above all when applied in bio-medical contexts and for body networked systems. Specific features of human body need to be taken into account in order to derive a biological propagation model for nanocommunications. Different communication alternatives have been considered, such as molecular and nano-communications [1], acoustic [2], molecular communications based on Forster Resonance Energy Transfer (FRET) phenomenon [3] just to mention some of them. A step towards the miniaturization at nanoscale level can be also moved by considering quantum phenomena. In fact, nanoscale cannot be considered as another step towards miniaturization, but it represents a kind of new scale [4]. Quantum phenomena can be considered at a purely quantum level or as derived from both quantum and classical laws and components. A specific type of quantum particles is represented by phonons, that represent the quanta of mechanical vibrations or quanta of lattice

Permission to make digital or hard copies of all or part of this work for personal or classroom use is granted without fee provided that copies are not made or distributed for profit or commercial advantage and that copies bear this notice and the full citation on the first page. Copyrights for components of this work owned by others than ACM must be honored. Abstracting with credit is permitted. To copy otherwise, or republish, to post on servers or to redistribute to lists, requires prior specific permission and/or a fee. Request permissions from permissions@acm.org.

NANOCOM'16, September 28-30, 2016, New York, NY, USA

(c) 2016 ACM. ISBN 978-1-4503-4061-8/16/09... $\$ 15.00$

DOI: http://dx.doi.org/10.1145/2967446.2967480 vibration (i.e. acoustic excitations). In practice, phonons represent quanta of ionic displacement field that describes classical sound waves.

In this paper, we will focus on a specific context, namely body network systems, where phonons can be generated by photon-phonon interaction. Specifically, we will characterize phonons as information carriers, and derive a theoretical expression of the information capacity. Furthermore, since we can assume that the biological information processing takes place at the challenging regime where quantum meets classical physics, we will focus on a specific biological scenario with a sample of human tissue irradiated with an electromagnetic field, representing the source generation of the photon-phonon interaction. The rest of the paper is organized as follow. In Section 2 we derive a theoretical evaluation of the capacity. In Section 3 we show a concrete example of biological tissue irradiated with an electromagnetic field and we show the capacity information in the specific case under analysis. Finally, conclusions and future directions are drawn at the end of the paper.

\section{INFORMATION CAPACITY}

In this paper we are interested to derive a theoretical information capacity in a phonon-based quantum network. For this aim, we start by considering the computation of the information capacity in a "traditional case". In a classical communication channel, the information capacity, namely the maximum number of bits that can be delivered in a reliable way, is given by considering the maximum of the Von Neuman entropy [5] as $S(\rho)=-\operatorname{Tr}\left[\rho \log _{2} \rho\right]$ where $\rho$ represents the input states.

If we assume that the entropy $S(\rho)$ is maximized on the states where the average energy is $E=\operatorname{Tr}[\rho H]$, and $H$ is the Hamiltonian, then we can solve the constrained maximization of $S(\rho)$ by using standard variational methods. This implies to solve the following equation:

$$
\delta\left\{S(\rho)-\frac{\lambda_{1}}{\ln 2} \operatorname{Tr}[H \rho]-\frac{\lambda_{2}}{\ln 2} \operatorname{Tr}[\rho]\right\}=0,
$$

where $\delta$ is the Dirac function, $\lambda_{1}$ and $\lambda_{2}$ are Lagrange multipliers that consider the normalization constraint $\operatorname{Tr}[\rho]=1$, and the $\ln 2$ factor is introduced to make possible the use of natural logarithms. Equation (1) can be solved by density matrix as $\rho=\frac{e^{-\beta H}}{Z(\beta)}$ where $Z(\beta)=\operatorname{Tr}\left[e^{-\beta H}\right]$ is the partition function of the system, and is determined by solving

$$
E=-\frac{\partial}{\partial(\beta)} \ln [Z(\beta)] .
$$


We can easy compute the capacity of the quantum channel as:

$$
C=S\left[\frac{e^{-\beta H}}{Z(\beta)}\right]=\frac{\beta E+\ln [Z(\beta)]}{\ln 2} .
$$

Of course, this represents an ideal capacity, since for real systems we must also account for intrinsic phonon losses, backscattering and re-thermalization effect.

From (1) we can argue that the evaluation of the system capacity can be done through the evaluation of its partition function $Z(\beta)$, that is generally not easy to be derived.

\section{PHONON CAPACITY IN A BIOLOGICAL CONTEXT}

In this section, we will examine the capacity of a phononbased quantum channel for biomedical applications, assuming an electromagnetic radiation is incident to a portion of human tissue.

Let us consider the photon-phonon interaction as mediated by a homogeneous sample of identical elements (namely, the human cells), which are in harmonic modes of vibration. We can consider a system of $n$ vibrating elements, each of them has an energy as

$$
E_{n}=\left(n+\frac{1}{2}\right) h f,
$$

where $E_{n}$ represents the energy of the $n$-th stationary state, $f$ is the frequency of a normal mode that an element vibrates accordingly to, and $h$ is the Planck's constant equal to $6.62606957 \times 10^{-34}[\mathrm{~J} \mathrm{~s}$.

When an element absorbs totally or partially a photon with an initial frequency $f_{i n}$, there is a phonon production that is regulated by Raman-Stokes scattering effect, and an excited phonon will have a frequency $f$. This results in the $n$-th element to acquire a higher energy, and the absorbed energy will be $E_{n}-E_{0}=n_{p} h f$, where $n_{p}$ represents the number of phonons. When a photon interacts inelastically with one element, it contributes to generate a random number of phonons with a randomized value of frequency. Notice that we are assuming the maximum number of phonons generated since we do not have prior information regarding the maximum number of phonons that can be created in a single collision between a photon and a cell. Furthermore, we assume that all the phonons will have the same frequency $f$. This means that the number of phonons will be exactly equal to the upper bound i.e., $\frac{k_{B} T}{h f}-\frac{1}{2}$.

Then, after a few calculations, it follows that in the case of wideband systems, the capacity can be derived as:

$$
C_{w b} \simeq \frac{\pi}{\ln 2} \sqrt{\frac{2 n+1}{3 \delta}} .
$$

In practice, we have determined that the capacity in the case of a homogeneous wideband channel is proportional to the number of phonons that are inversely proportional to the frequency. In the specific case of a biological tissue, there exists a frequency band in the range $\left[10^{3}, 10^{12}\right] \mathrm{Hz}$, where the energy absorbed reaches its maximum value and it seems that there is no appreciable absorption beyond this band. So, with the aim of characterizing the phonon-based quantum channel capacity, we considered this frequency range.

In Figure 1 we show the capacity of the system expressed in [qubit/s] by varying the frequency in the range $\left[10^{3}, 10^{12}\right] \mathrm{Hz}$.

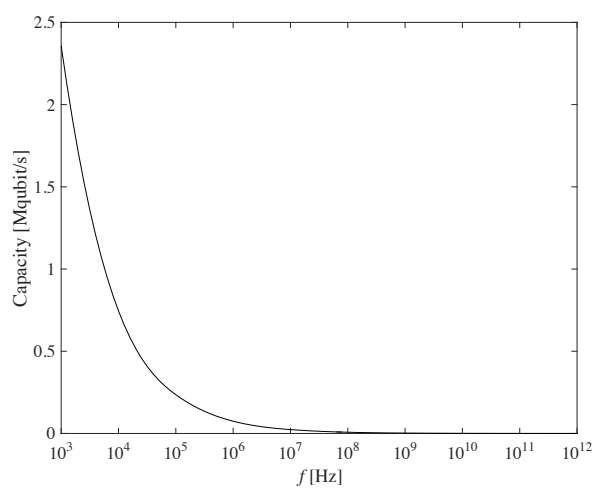

Figure 1: Capacity of phonon-based quantum channel versus frequency.

Since the number of phonons, at a fixed temperature, is higher at lower frequency modes, the capacity will be higher at lower frequencies. It is worth to recall that we are considering a $1-t o-1$ correspondence in the phonon generation from one incident photon. This means that for each photon generated there will be one phonon.

\section{CONCLUSIONS}

In this paper we analyzed specific quantum particles, named phonons, representing acoustic excitations, from a communication perspective. Specifically, we assumed phonons as potential information carriers. Through the radiation of an electromagnetic source on a biological tissue, we observe the generation of a photon-phonon interaction, that allows to derive the theoretical information capacity of these generated phonons.

The work dealt in this paper has as objective to start the characterization of a phonon-based networked system, that could be exploited in a future for addressing the design of nano-motors with specific features matching the environment under analysis. As future investigations, we aim to refine the model presented by taking into account of real phenomena.

\section{REFERENCES}

[1] I. F. Akyildiz, J.M. Jornet, and M. Pierobon. Nanonetworks: A new frontier in communications. Communications of the ACM, 54(11):84-89, 2011.

[2] V. Loscrí and A.M. Vegni. An acoustic communication technique of nanorobot swarms for nanomedicine applications. IEEE Transaction on Nanobioscience, 14(6):598-607, 2015.

[3] M. Kuscu and O.B. Akan. Coverage and throughput analysis for fret-based mobile molecular sensor/actor nanonetworks. Elsevier Nano Commmunication Networks, 5:45-53, 2014.

[4] I.F. Akyildiz, J.M Jornet, S. Balasubramanian, and Y. Koucheryavy. Internet of bionanothings. IEEE Communications Magazine, 53(3):32-40, 2015.

[5] V. Giovannetti, S. Lloyd, and L. Maccone. Capacity of nonlinear bosonic systems. Physical Review A, 70:012307-1-012307-6, 2004. 